\title{
Flow, flow transition and runout distances of flowing avalanches
}

\author{
B. SALM \\ Eidgenössisches Institut für Schnee- und Lawinenforschung, CH-7260 Weisffluhjoch/Davos, Switzerland
}

\begin{abstract}
A simple quasi one-dimensional model of flowing avalanches is presented. It is a further development of that used in the Swiss Guidelines for practitioners. It is shown that shearing in avalanche movement is concentrated near the ground and that, due to the geometrical roughness of the ground, a flow resistance proportional to the square of velocity must be taken into account in addition to dry friction. For the change of flow on changing slope angles it is demonstrated that under certain conditions for internal friction a "normal" flow on a flat lower part can no longer be attained; the avalanche behaves like a rigid body. The runout distance is in fair agreement with the Guidelines if a larger internal friction is used. The main differences lie in much smaller deposition depths and smaller velocities during runout.
\end{abstract}

\section{INTRODUCTION}

In Switzerland a simple model for flowing avalanches is widely used for practical purposes (Salm and others, 1990). This model — designated as Guidelines — is based on theoretical considerations, field measurements of velocities, and examination of several hundred observed avalanches, especially runout distances. Further developments will be presented and existing relations are specified more precisely. As the flow depth of an avalanche is much smaller than its length, a quasi onedimensional flow is assumed.

\section{FLOW PROFILE}

The main question here is the distribution of shearing within the moving snow, i.e. internal shearing within the avalanching snow versus shearing near the ground (sliding). The only measurement in a natural avalanche by Gubler and others (1986) shows a shear concentration very close to the ground and nearly no internal shearing.

Moving snow in natural avalanches seems to be not a homogeneous but a structured mass. Even in a more or less steady movement, field tests (e.g. Schaerer and others, 1980) show not constant but oscillating impact pressures with pronounced peaks in rapid sequences. This can be interpreted as an effect of lumps of snow much larger than single snow grains.

We consider, therefore, avalanching snow as consisting of clods of snow, idealized as spheres with a uniform diameter, $\phi$, of an order of magnitude of $0.1 \mathrm{~m}$. Contact forces between spheres are assumed to be dry friction, characterized by the internal friction angle, $\varphi$, and impact forces with a coefficient of restitution $e \sim 0$ (i.e. snow and not single ice grains). Dry friction acts in the contact plane and impact forces perpendicular to it. The driving force is gravity.

Roughly seen, an internal movement parallel to the slope is only possible if $\varphi$ is smaller than the slope angle, $\psi$. Internal friction has never been measured directly, but from observations of avalanche deposits and measurements by Roch (1966), $\varphi$ can be estimated at least to be about $25^{\circ}$, i.e. about the slope of avalanche tracks. Therefore an internal shearing movement of the overburden seems improbable, especially in the runout zone where $\psi \leq 15^{\circ}$.

Sliding near ground seems to be the decisive process determining avalanche speed. To get a qualitative insight, a simple model analogous to that of Bagnold $(1954,1966)$ is used (Salm, unpublished a). To estimate the flow resistance due to impact of the moving snow, the roughness of the ground is represented by obstacles protruding from the ground with a mean distance of $\Delta r$ in flow direction $x_{1}$ (Figure 1). The heights of these obstacles are at least of the same order of magnitude as the clods of snow. Flow depth, $d$, and coordinate $x_{2}$ are perpendicular to the slope.

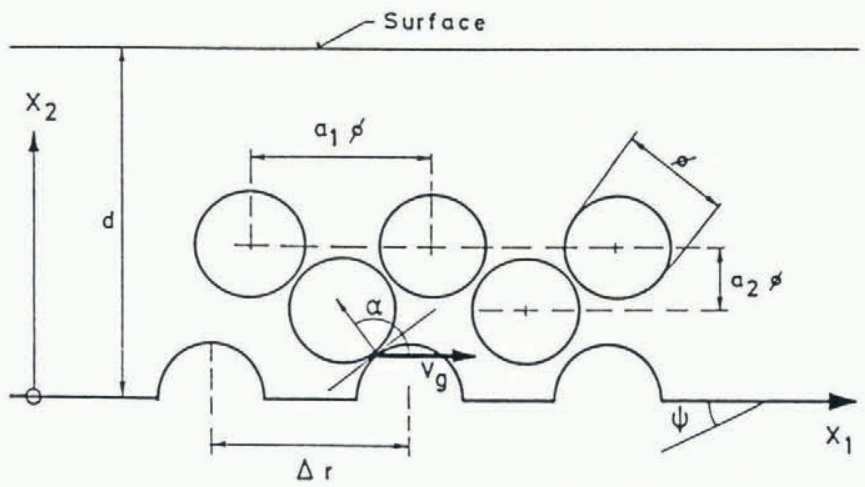

Fig. 1. Model for shearing near the ground. 
If the speed over ground is $v_{\mathrm{g}}$, then $v_{\mathrm{g}} / \Delta r$ collisions in unit time occur. The change of momentum in $x_{1}$ direction per sphere with $e=0$ is $m v_{\mathrm{g}} \cos ^{2} \alpha$. The impact angle, $\alpha$, is between $v_{\mathrm{g}}$ and the normal of the contact surface and $m$ is the mass of a particle with density $\rho_{8}$ of snow. The number of spheres per unit area parallel to ground is $\left(a_{1} \phi\right)^{-2}$

The total shear resistance due to impact is then

$$
\tau \sim\left(a_{1} \phi\right)^{-2} \frac{v_{\mathrm{g}}}{\Delta r} m v_{\mathrm{g}} \cos ^{2} \alpha .
$$

Introducing the mass of a sphere $\rho_{\mathrm{s}} \pi \phi^{3} / 6$ and the number of spheres per unit volume $\left(a_{1}^{2} a_{2} \phi^{3}\right)^{-1}$ we get for the mean density of flowing snow

$$
\frac{\rho_{\mathrm{m}}}{\rho_{\mathrm{s}}}=\left(a_{1}^{2} a_{2}\right)^{-1} \pi / 6,
$$

and therefore

$$
\tau \sim\left(\frac{g \Delta r}{a_{2} \phi \cos ^{2} \alpha}\right)^{-1} \rho_{\mathrm{m}} g v_{\mathrm{g}}{ }^{2}=\frac{\rho_{\mathrm{m}} g}{\xi} v_{\mathrm{g}}{ }^{2} .
$$

This is the well known "turbulent" friction, first stated by Voellmy (1955). He, however, introduced this term in analogy to turbulent open-channel flow in hydraulics (Chézy equation).

The above relations are complicated by the fact that $a_{1}, a_{2}$ and $\alpha$ are functions of speed (personal communication from J. Dent), so that $\rho_{\mathrm{m}}$ and $\xi$ become velocity-dependent too.

It is to be expected that $\alpha\left(180^{\circ} \geq \alpha \geq 90^{\circ}\right)$ decreases with higher velocities. Furthermore, the terrain roughness will be smoothed to a certain degree by deposited snow, depending on obstacle heights.

If $\tau$ of Equation (2) decreases to zero (for $\alpha=90^{\circ}$ or $\Delta r=\infty$ ) only dry friction on ground remains as resistance. The coefficient of dry friction, $\mu$, of sliding is well known from observations in runout zones. Its value $0.155 \leq \mu \leq$ 0.30 is much less than that of $\tan \varphi$ (Salm and others, 1990). Adding this dry friction to the "turbulent" friction yields

$$
v_{\mathrm{g}}=(\xi d)^{\frac{1}{2}}(\sin \psi-\mu \cos \psi)^{\frac{1}{2}} .
$$

Observations of real avalanches (Gubler and others, 1986) are at least not in contradiction with the described concept. A careful inspection of these data shows that at least two resistances must occur. In a fracture zone with smooth terrain, dry friction dominates and leads to high accelerations. As soon as rugged terrain is reached the resistance increases, obviously due to an additional velocity-dependent force.

It can be concluded that the velocity profile of a flowing avalanche can be idealized by a rectangular shape with Equation (3) as average velocity. (This holds as long as the slope is not too steep, say $\psi<35^{\circ}$.) The coefficient $\mu$ is mainly connected to snow properties, whereas $\xi$ depends mainly on terrain geometry. If the roughness is not large, $\xi$ increases with speed so that asymptotically only dry-friction resistance remains (personal communication from J. Dent).

\section{FLOW TRANSITION}

We assume that over a limited time the discharge, $Q$ $\left(\mathrm{m}^{3} \mathrm{~s}^{-1}\right)$, in a flowing avalanche is constant (Salm, 1972). With a homogeneous and constant density $\left(\rho_{\mathrm{m}}=\rho\right)$ and with a nearly one-dimensional movement $(\partial d / \partial x<<1)$ we have

$$
Q=B d v=\text { constant },
$$

where $B$ is the width of the avalanche.

On a uniform slope angle $\psi_{0}$, the velocity with Equations (3) and (4) is

$$
v_{0}^{3}=\frac{Q}{B} \xi\left(\sin \psi_{0}-\mu \cos \psi_{0}\right) .
$$

As soon as $\psi_{0}$ changes to $\psi_{u}$ in a point $\mathrm{A}$, the velocity changes gradually from $v_{0}$ to $v_{u}$, and with this the flow depth from $d_{0}$ to $d_{u}$.

We consider a volume element on the slope $\psi_{u}$ fixed in the space. Its length in flow direction $x_{1}$ is $d$ and we assume a quasi one-dimensional movement in the $x_{1}-x_{2}$ plane.

We start from the global form of momentum theorem,

$$
\int \frac{\partial}{\partial t}\left(\rho v_{k}\right) \mathrm{d} V=\int\left(\sigma_{j k}-\rho v_{j} v_{k}\right) \nu_{j} \mathrm{~d} F+\int \rho K_{k} \mathrm{~d} V
$$

with time, $t$, volume, $V$, surface, $F$, stress, $\sigma_{j k}$, unit vector, $\nu_{j}$ (with positive direction to the outer side of the volume), and with the body force, $K_{k}$.

In the plane $x_{2}-x_{3}$ perpendicular to the flow direction $x_{1}$ we have the resultant pressure force of $\lambda \rho g d^{2} / 2$ with

$$
\lambda=\tan ^{2}\left(45^{\circ}+\frac{\varphi}{2}\right)
$$

if $\psi_{0}>\psi_{u}$ (passive snow pressure). This represents the upper limit of the force, corresponding to the ColombMohr criterion. (In a flow on an infinite slope, $\lambda$ must be about unity.)

If a steady movement is assumed, the following expressions are obtained (with $x_{1}=x$ and $v_{1}=v$ ):

$$
\begin{aligned}
\int \sigma_{11} \nu_{1} \mathrm{~d} F & =-\lambda \rho g d^{2} \frac{\partial d}{\partial x} \\
\int \sigma_{21} \nu_{2} \mathrm{~d} F & =-\rho g \mu \cos \psi_{u}\left(1+\frac{1}{2} \frac{\partial d}{\partial x}\right) d^{2}-\frac{\rho g}{\xi}\left(v^{2}+v \frac{\partial v}{\partial x} d\right) d \\
\int \rho v_{1}^{2} \nu_{1} \mathrm{~d} F & =\rho d^{2} 2 v \frac{\partial v}{\partial x}+\rho d v^{2} \frac{\partial d}{\partial x} \\
\int \rho K_{1} \mathrm{~d} V & =\rho g\left(d+\frac{d}{2} \frac{\partial d}{\partial x}\right) d \sin \psi_{u},
\end{aligned}
$$

and from this the differential equation

$$
\mathrm{d} x=a \frac{b+c u^{3}}{u^{2}-u^{5}} \mathrm{~d} u
$$

with the dimensionless velocity $u=v / v_{u}$ and

$$
\begin{aligned}
a & =d_{u} \xi / g \\
b & =\frac{g}{\xi}\left(\frac{1}{2}-\frac{\lambda}{\sin \psi_{u}-\mu \cos \psi_{u}}\right) \\
c & =1+g / \xi .
\end{aligned}
$$




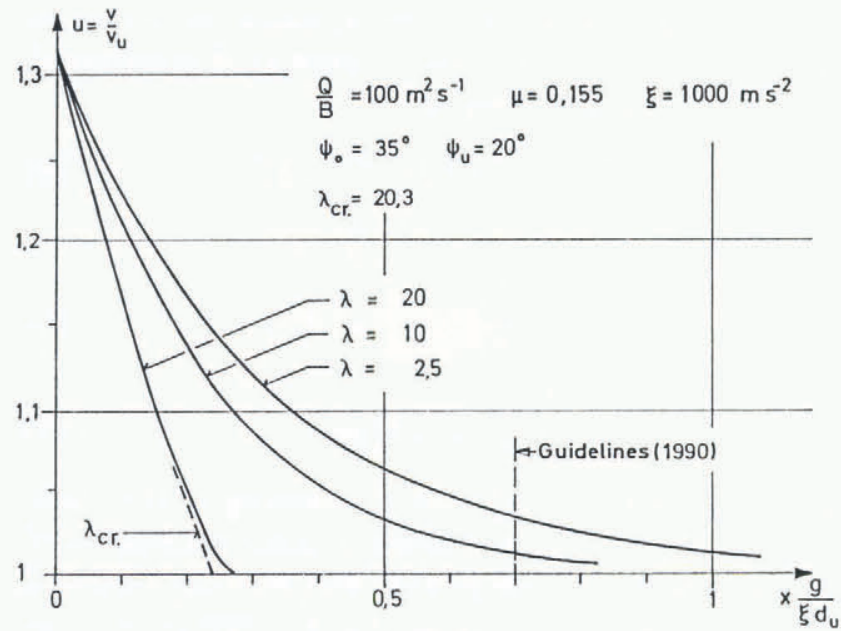

Fig. 2. Flow transition for different values of $\lambda$.

With the initial condition $u_{0}=v_{0} / v_{u}$ for $x=0$ the solution is

$$
\begin{aligned}
\frac{x}{a}= & b\left(\frac{1}{u_{0}}-\frac{1}{u}\right)+(b+c)\left[\frac{1}{6} \ln \frac{\left(1-u_{0}\right)^{2}}{1+u_{0}+u_{0}^{2}} \cdot \frac{1+u+u^{2}}{(1-u)^{2}}\right. \\
& \left.+\frac{1}{\sqrt{3}}\left(\tan ^{-1} \frac{2 u_{0}+1}{\sqrt{3}}-\tan ^{-1} \frac{2 u+1}{\sqrt{3}}\right)\right] .
\end{aligned}
$$

The "normal" flow on $\psi_{u}$ with $u=1$ will theoretically be attained as $x \rightarrow \infty$.

Figure 2 shows an example for the decrease of $u$ for a flow from steeper to flatter terrain with different values of $\lambda$. This decrease is faster for larger $\lambda$ because of the larger passive snow pressure on the flat part. There is however a restriction: if $\lambda$ is too large "normal" flow on $\psi_{u}$ cannot be attained. The limiting value for $\lambda$ can be seen from Equation (8). If $b+c=0$ then

$$
\lambda_{\text {cr. }}=\left(\frac{3}{2}+\frac{\xi}{g}\right)\left(\sin \psi_{u}-\mu \cos \psi_{u}\right) \text {. }
$$

For larger values than Equation (9) the flow on $\psi_{u}$ continues with $v_{0}$ and $d_{0}$. The driving force $\rho g d_{u}\left(\sin \psi_{u}-\mu \cos \psi_{u}\right)$ - too small to maintain a steady flow - is enlarged by the passive snow pressure at point A given by $d_{0}$. After a critical length, this pressure will be too small to maintain this "deficit" flow; $d_{0}$ will be gradually enlarged to $d_{u}$ so that "normal" flow is established on $\psi_{u}$.

The assumed parameters for $\mu$ and $\xi$ in Figure 2 are empirical ones from observed avalanches as given in the Guidelines (Salm and others, 1990). From Equation (2) a value for $\xi=1000 \mathrm{~m} \mathrm{~s}^{-2}$ would be obtained for $a_{2}=0.8$, $\Delta r / \phi=10$ and $\alpha=110^{\circ}$.

The steepest gradient of $u$ in Figure 2 occurs for $x=0$ and $\lambda=20$ which gives $\partial d / \partial x=10^{-2}$. The assumption of a quasi one-dimensional flow is therefore fullfilled.

The dimensionless quantity in Equation (8),

$$
\frac{x}{a}=\frac{g}{\xi d_{u}} x=\frac{g B v_{u}}{\xi Q} x
$$

is used in practical calculations to decide whether or not "normal" flow is reached on a certain slope after a certain length $x$. In the Guidelines a value of 0.7 is given after which the velocity differs less than $10 \%$ from "normal" flow conditions. This value has been calculated earlier by Salm (unpublished b) with a simpler approach.
The influence of $\lambda$ has been neglected and $g / \xi$ was assumed to be very small, so that $b=0$ and $c=1$, which delivers the differential equation

$$
\mathrm{d} x=a \frac{u}{1-u^{3}} \mathrm{~d} u
$$

with a solution similar to Equation (8). Figure 2 shows that 0.7 is the maximum for $\lambda \geq 10$. It is however too small for $\lambda=2.5$, a value which is probably not realistic for a pressure zone (see section below).

To avoid difficulties in estimating the transition zone with a simple rule, Equation (8) could be used directly to calculate the velocities on limited sections with constant slope, however with considerable effort.

\section{RUNOUT DISTANCE}

The situation is similar to that with flow transition: at a point $P$ the slope angle changes from $\psi_{0}$ to $\psi_{u}$, however for $\psi_{u}$ the condition

$$
\tan \psi_{u}=\tan \psi_{\mathrm{s}}<\mu
$$

must be fulfilled, i.e. not a driving but a retarding force must occur. This retarding force is originating from the velocity-independent dry friction, which is the only effective mechanism to slow down the movement to standstill on a slope inclined in direction of the speed. This is the runout situation observed for most avalanches. It may be remarked that Equation (10) allows a very simple measurement of the minimum acting friction coefficient of observed avalanches.

Again the mean density $\rho_{\mathrm{m}}=\rho$ is considered constant, and the length of the runout is about two orders of magnitude larger than $d$, so that again a quasi onedimensional movement can be assumed.

The supply of avalanching snow at point $P$ is given by $Q$, which is constant for the time between the entrance of the snow at $P$ until the standstill.

The velocity of the snow mass between the front and $P$ is in every point constant but time-dependent. This can be justified with the often observed "train-like" behaviour in the runout: the movement seems similar to that of a rigid body. The reason for this lies in high internal friction angles plus (probably) a cohesion which may be created quickly in relatively slow-moving snow in flat terrain (Gubler, 1982).

The depth, $d$, in the runout is larger than the flow depth of the entering snow, $d_{P}$, at $P$. This difference, $\Delta d$, is due to the velocity difference of $v_{P}$ at $P$ and $v$ of the retarding snow mass, and can be calculated approximately from the kinetic and pressure energy in analogy to hydraulics:

$$
\frac{v_{P}^{2}}{2 g}+\lambda d_{P}=\frac{v^{2}}{2 g}+\lambda\left(d_{P}+\Delta d\right)
$$

and yields

$$
\Delta d \cong \frac{v_{P}^{2}-v^{2}}{2 g \lambda} .
$$

For our calculation we will assume a constant mean depth, $d_{\mathrm{m}}$, over the final runout distance, $x_{\mathrm{s}}$. A first assumption of $d_{\mathrm{m}}$ will allow us to calculate $v$ and then to 
check this assumption and, if necessary, to correct it. Furthermore, it will be possible to check the total supply of mass at $P$ during the time interval until standstill, and then this mass can be compared with that obtained from Equation (11). These problems will be discussed later; at present we assume all kinetic energy from the velocity difference at $P$ is completely transferred into an increase of depth.

In the avalanche front the depth cannot be increased immediately; it will remain constant, equal to $d_{P}$ over a certain time or length. This length, $\Delta$, is attained as soon as the increasing snow pressure at $P$ arrives at its maximum, $\lambda \rho g d_{P}{ }^{2} / 2$, and is in equilibrium with flow resistance and driving force; thus

$$
\Delta=\frac{\lambda d_{P}^{2} / 2}{d_{P}\left(\mu \cos \psi_{\mathrm{s}}-\sin \psi_{\mathrm{s}}\right)+v_{P^{2}} / \xi} .
$$

The theorem of momentum is formulated for unity width $B$. The distance of $P$ to the avalanche front is $x+\Delta$ and the mass is

$$
M=\rho d_{\mathrm{m}}(x+\Delta) .
$$

$$
\frac{\partial}{\partial t}(M v)=\rho d_{\mathrm{m}}(x+\Delta) \frac{\partial v}{\partial t}+v \rho d_{\mathrm{m}} \frac{\partial x}{\partial t}=\text { Resultant force. }
$$

The term $\partial d / \partial t$ is neglected and $\partial v / \partial t=v \partial v / \partial x$.

$$
\begin{aligned}
& \text { Resultant force }=-\lambda \rho g \frac{d_{P}^{2}}{2}-\rho g(x+\Delta) d_{\mathrm{m}} \\
&\left(\mu \cos \psi_{\mathrm{s}}-\sin \psi_{\mathrm{s}}\right)-\frac{\rho g}{\xi}(x+\Delta) v^{2} .
\end{aligned}
$$

This leads to the differential equation

$$
\begin{aligned}
\frac{\partial v}{\partial x}=-\left(\frac{1}{x+\Delta}+\frac{g}{\xi d_{\mathrm{m}}}\right) v & +\left[\frac{\lambda g d_{P}{ }^{2}}{2 d_{\mathrm{m}}(x+\Delta)}\right. \\
& \left.-g\left(\mu \cos \psi_{\mathrm{s}}-\sin \psi_{\mathrm{s}}\right)\right] .
\end{aligned}
$$

With the initial condition $v=v_{P}$ for $x=0$ we get the solution

$$
\begin{aligned}
v^{2} & =\frac{a^{\prime} / b^{\prime}}{(x+\Delta)^{2}}\left[\left(x-\frac{1}{b^{\prime}}\right)\left(\frac{c^{\prime}}{a^{\prime}}-2 \Delta\right)-\left(x-\frac{1}{b^{\prime}}\right)^{2}\right. \\
& -\frac{1}{b^{\prime 2}}+\Delta\left(\frac{c^{\prime}}{a^{\prime}}-\Delta\right)+\mathrm{e}^{-b^{\prime} x}\left\{v_{P}^{2} \frac{\Delta^{2}}{a^{\prime} / b^{\prime}}+\left(\Delta-\frac{1}{b^{\prime}}\right)^{2}\right. \\
& \left.\left.+\frac{1}{b^{\prime 2}}-\frac{c^{\prime}}{a^{\prime}}\left(\Delta-\frac{1}{b^{\prime}}\right)\right\}\right]
\end{aligned}
$$

with

$$
\begin{aligned}
& a^{\prime}=2 g\left(\mu \cos \psi_{\mathrm{s}}-\sin \psi_{\mathrm{s}}\right) \\
& b^{\prime}=2 g /\left(\xi d_{\mathrm{m}}\right) \\
& c^{\prime}=\lambda g d_{P}{ }^{2} / d_{\mathrm{m}}
\end{aligned}
$$

and furthermore

$$
a^{\prime} / b^{\prime}=\xi d_{\mathrm{m}}\left(\mu \cos \psi_{\mathrm{s}}-\sin \psi_{\mathrm{s}}\right)=V^{2} .
$$

A solution exists only with a positive $V^{2}$, thus runout condition (10) is fulfilled. For $x \rightarrow \infty$ we get $v^{2} / V^{2}=-1$.

The total runout distance is $x_{8}=x_{0}+\Delta$, where $x_{0}$ corresponds to $v^{2}=0$ in (14) and $\Delta$ is taken from (12).

The calculation of the runout distance in the Guidelines (1990) is based on Salm (unpublished b). There only the runout of the constant front mass, $\rho d_{\mathrm{m}} \Delta$, is considered and the influence of the snow pressure at $P$ is neglected. For the velocity we arrived at

$$
v^{2}=\left(v_{P}^{2}+V^{2}\right) \mathrm{e}^{-b^{\prime} x}-V^{2},
$$

and for runout distance

with

$$
x_{\mathrm{sG}}=\frac{1}{b^{\prime}} \ln \left(1+v_{P}^{2} / V^{2}\right),
$$

$$
d_{\mathrm{mG}}=d_{P}+v_{P}^{2} / 4 g \lambda .
$$

The subscript $G$ denotes values according to the Guidelines. The latter relation assumes a linear decrease of $v^{2}$ with $x$, based on the assumption that only dry friction is acting. The coefficient $\lambda$ is fixed at 2.5. With the above formulas, and mainly with observed runout distances, the coefficients $\mu$ and $\xi$ were calibrated. The agreement is satisfactory although the applied $d_{\mathrm{m}}$ in (17) with $\lambda=2.5$ seems to furnish too large values.

To compare the calibrated rules (15)-(17) with (12) and (14), the following examples have been calculated:

friction coefficients: $\mu=0.155 \xi=1000 \mathrm{~m} \mathrm{~s}^{-2}$;

discharge: $Q / B=10 / 50 / 100 / 200 \mathrm{~m}^{2} \mathrm{~s}^{-1}$;

slope angle before $P$ : $\psi_{P}=25^{\circ}$;

slope angle in the runout zone: $\psi_{8}=0^{\circ} / 7.5^{\circ}$.

The agreement of $x_{s}$ from Equations (12) and (14) with $x_{\mathrm{sG}}$ is surprisingly good, but only if the large value of $\lambda=20\left(\varphi=65^{\circ}\right)$ is introduced. The ratio $x_{\mathrm{s}} / x_{\mathrm{sG}}$ is 1.02 and 1.18 for $\psi=0^{\circ}$ and $7.5^{\circ}$ respectively, which means that for slopes $\psi_{\mathrm{s}}$ close to the critical value of $\tan ^{-1} \mu=8.8^{\circ}$ the runout distance becomes larger due to the snow pressure at $P$.

For $x_{\mathrm{s}}$ the mean deposition depth $d_{\mathrm{m}}$ is 2.5-2.8 times smaller than for $x_{\mathrm{sG}}$, according to observations a reasonable result.

The mean value $d_{\mathrm{m}}$ was checked with Equations (14) and (11). The mean square of the velocity from (14) is

$$
x_{\Delta}\left(v^{2}\right)_{\mathrm{m}}=\int_{\Delta}^{x_{\Delta}} v^{2} \mathrm{~d} x+\Delta v_{P}^{2}
$$

and

$$
d_{\mathrm{m}}=d_{P}+\Delta d=d_{P}+\left[v_{P}^{2}-\left(v^{2}\right)_{\mathrm{m}}\right] /(2 g \lambda) .
$$

A good estimation for $\Delta d$ is $v_{P}^{2} /(3.94 g \lambda)$ and $v_{P}^{2} /(2.50 g \lambda)$ for $\psi_{8}=0^{\circ}$ and $7.5^{\circ}$ respectively. For different values of $\psi_{\mathrm{s}}$ a linear interpolation will furnish satisfactory results.

Considering the conservation of mass we assume a constant supply $Q / B=v_{P} d_{P}$ from the moment when the front passes $P$ until it reaches $x_{8}$, therefore

$$
x_{\mathrm{s}} v_{\mathrm{m}}=\int_{\Delta}^{x_{\mathrm{t}}} v \mathrm{~d} x+\Delta v_{P},
$$

and with this the average depth from supply

$$
d_{\mathrm{ms}}=\left(v_{P} d_{P}\right) / v_{\mathrm{m}} .
$$

In Figure 3 the different depths, $d_{\mathrm{m}}, d_{\mathrm{ms}}$ and $d_{\mathrm{mG}}$, are plotted as a function of $Q / B$ and the two slope angles, $\psi_{8}$, of $0^{\circ}$ and $7.5^{\circ}$.

It is clear that the depth from supply must be equal to or larger than the dynamic depth of (17) or (18). As it can be seen from Figure $3, d_{\mathrm{ms}}$ is always larger than $d_{\mathrm{m}}$. 


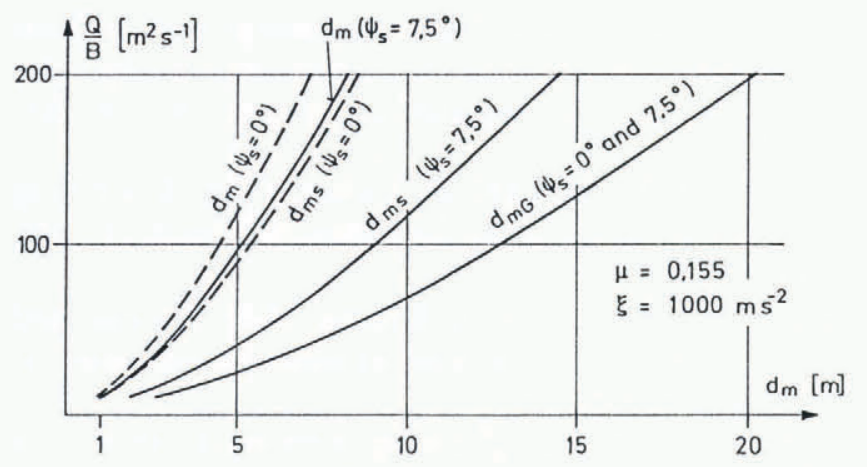

Fig. 3. Mean deposition depth of the present calculation $d_{\mathrm{m}}$, the supply of mass $d_{\mathrm{ms}}$ and of the Guidelines.

Obviously not all of the supplied mass can be drained off below $P$. What happens with the extra mass given by $d_{\mathrm{ms}}-d_{\mathrm{m}}$ ? It must be taken into account that above $P$ a back-pressure zone is gradually building up. (Before the front reaches $P, \lambda$ of the avalanching must be of the order of one.) This will slow down the speed and increase $d$, and finally a part of the mass will remain above $P$. If the avalanche is longer than is assumed here, the extra mass could be considerably larger and will be deposited above the mass at rest.

In Figure 3 it can furthermore be seen that the mean deposition depth, $d_{\mathrm{mG}}$, is indeed too large: the supplied mass cannot fill up the required deposition!

Finally in Figure 4 an example is given of an observed avalanche (Samedan/Ariefa on 2 January 1951). The plot of $v^{2}$ versus $x$ shows, with calculation according to (14), a surprisingly large gradient $\partial v^{2} / \partial x$ after the mass of length $\Delta$ has passed. The deceleration is much stronger compared to that of (15). This is an effect of the increasing mass which increases the flow resistance gradually, in contrast to (15) which considers only a constant front mass. The sharp change of the gradient after passage of the distance $\Delta$ will cause a large increase of $\Delta d$ in the front part and therefore a steep deposition front. This is in accordance with observations. Velocity measurements of Gubler and others (1986) also show steep gradients, $\partial v / \partial x$, in the runout zone (e.g. Mader Grond avalanche on 17 January 1985), but it was

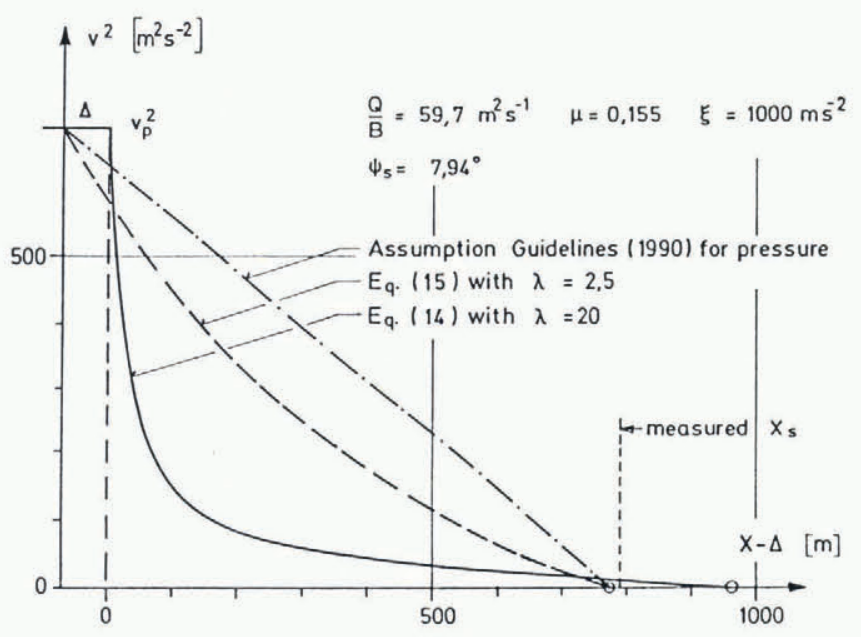

Fig. 4. Distribution of $v^{2}$ over the runout zone. impossible to determine the exact position of this deceleration.

The distribution of the pressure $p=\rho v^{2}$ on an obstacle in the runout zone is important for the elaboration of avalanche-danger maps, because this is, in addition to the return period, a criterion for the degree of danger. In the Guidelines (1990) a linear decrease of $v^{2}$, and therefore also of $p$, is assumed. With this, one is in any case on the safe side.

The observed runout distance of the Samedan/Ariefa avalanche was $860 \mathrm{~m}$. On the basis of climatological data and a guess of additional local wind influence, a mean fracture depth $d_{0}=1.00 \mathrm{~m}$ in the starting zone was assumed. Equation (16) delivers a runout distance of $844 \mathrm{~m}$, whereas Equation (14) gives a somewhat too large value of $1031 \mathrm{~m}$. However, if a slightly less pessimistic wind influence is assumed, we can just assume $d_{0}=0.8 \mathrm{~m}$. In this case equation (16) delivers $x_{\mathrm{sG}}=720 \mathrm{~m}$ and Equation (14) $807 \mathrm{~m}$ ! This demonstrates that the runout distance is very sensitive to fracture depth, a quantity which is very difficult to determine locally. Unfortunately so far no accurate measurements on the whole fracture area (e.g. by photogrammetry) before and after an avalanche event have been made.

\section{CONCLUSIONS}

A rectangular velocity profile can be justified, because shearing is concentrated in the bottom layer. In addition to dry friction, a flow resistance proportional to the square of speed must be taken into account, because of the plastic behaviour of the snow clods and the uneven ground.

The recommendation of the Guidelines for a transition zone of $0.7 \mathrm{~g} /\left(\xi d_{u}\right)$, when the slope changes from $\psi_{0}$ to $\psi_{u}$, can be maintained. If however a critical value, $\lambda$, of the snow is exceeded, a "normal" flow on $\psi_{u}$ cannot be attained. The flow on $\psi_{u}$ will continue, over a limited distance, corresponding to a speed according to $\psi_{0}$. This will usually occur for values of $\psi_{u}$ close to the critical value of $\tan ^{-1} \mu$.

The new model for runout - taking into account an increasing mass and snow pressure due to $\lambda$ - is in fairly good agreement with the calculation according to the Guidelines if a high value for $\lambda$ is taken. For values of $\psi_{\mathrm{s}}$ close to the critical angle $\tan \psi_{\text {cr. }}=\mu$, the runout distance is about $20 \%$ larger in the new model. The main differences from the Guidelines are a much lower mean deposition depth and a much faster decrease of velocity. The instructions given in the Guidelines by Equations (16) and (17) can be maintained, although if real depths are needed, the proposed lower values should be applied. The velocity distribution, used for the pressure distribution in avalanche-hazard maps, could be replaced by a faster decrease than linearly with the square of speed.

\section{REFERENCES}

Bagnold, R. A. 1954. Experiments on a gravity-free dispersion of large solid spheres in a Newtonian fluid under shear. Proc. R. Soc. London, Ser. A, 225, 49-63. 
Bagnold, R. A. 1966. The shearing and dilatation of dry sand and the "singing" mechanism. Proc. $R$. Soc. London, Ser. A, 295, 219-232.

Gubler, H. U. 1982. Strength of bonds between ice grains after short contact times. F. Glaciol., 28(100), 457-473.

Gubler, H., M. Hiller, G. Klausegger and U. Suter. 1986. Messungen an Fliesslawinen. Eidg. Inst. Schnee- Lawinenforsch. Mitt. 41.

Roch, A. 1966. Les variations de la résistance de la neige. International Association of Scientific Hydrology Publication 69 (Symposium at Davos 1965 - Scientific Aspects of Snow and Ice Avalanches), 86-99.

Salm, B. 1972. Grundlagen des Lawinenverbaues. Bündnerwald, Beiheft 9, 67-81.

Salm, B. Unpublished a. Das Geschwindigkeitsprofil in einer Fliesslawine. Eidgenössisches Institut für Schneeund Lawinenforschung. Interner Bericht 574, 1979.
Salm, B. Unpublished b. Fliessübergänge und Auslaufstrecken von Lawinen. Eidgenössisches Institut für Schnee- und Lawinenforschung. Interner Bericht 566, 1979.

Salm, B., A. Burkard and H. Gubler. 1990. Berechnung von Fliesslawinen. Eidg. Inst. Schnee- Lawinenforsch. Mitt. 47.

Schaerer, P. A. and A. A. Salway. 1980. Seismic and impact-pressure monitoring of flowing avalanches. 7 . Glaciol., 26(94), 179-187.

Voellmy, A. 1955. Über die Zerstörungskraft von Lawinen. Schweiz. Bauztg., 73, 159-165, 212-217, 246-249, 280-285.

The accuracy of references in the text and in this list is the responsibility of the author, to whom queries should be addressed. 